\title{
Transmission-blocking activity of antibodies to Plasmodium falciparum GLURP.10C chimeric protein formulated in different adjuvants
}

Will Roeffen ${ }^{1 *}$, Michael Theisen ${ }^{2,3,4}$, Marga van de Vegte-Bolmer ${ }^{1}$, GeertJan van Gemert ${ }^{1}$, Theo Arens ${ }^{1}$, Gorm Andersen ${ }^{2}$, Michael Christiansen², Laxman Sevargave ${ }^{5}$, Shrawan Kumar Singh ${ }^{5}$, Swarnendu Kaviraj ${ }^{5}$ and Robert Sauerwein ${ }^{1}$

\begin{abstract}
Background: Plasmodium falciparum is transmitted from person to person by Anopheles mosquitoes after completing its sexual reproductive cycle within the infected mosquito. An efficacious vaccine holds the potential to interrupt development of the parasite in the mosquito leading to control and possibly eradication of malaria. A multi-component, R0.10C, was developed comprising P. falciparum glutamate-rich protein (R0) fused in frame to a correctly folded fragment of Pfs $48 / 45$ (10C). Here, a series of novel adjuvants were screened for their ability to elicit transmissionblocking (TB) antibodies.

Methods: The recombinant fusion protein R0.10C was produced in Lactococcus lactis and purified by affinity-chromatography on a monoclonal antibody (mAb 85RF45.1) against a major epitope for TB antibodies (epitope 1) harboured on R0.10C. Immune-purified R0.10C was mixed with a series of adjuvants and tested in mice and rats.

Results: In general, all R0.10C formulations elicited high levels of antibodies recognizing native Pfs48/45 in macrogametes/zygotes. TB activity of anti-R0.10C antisera was assessed in the standard membrane-feeding assay (SMFA). Potency of different adjuvant/R0.10C combinations was tested in mice and rats using aluminium hydroxide (Alum), Alum with micellar and emulsion formulations of a synthetic TLR4 agonist, Glucopyranosyl Lipid Adjuvant (GLA), stable emulsion (SE)/GLA, AbISCO-100 and Freund's adjuvant (as reference). All formulations produced high antibody titres recognizing the native Pfs $48 / 45$ protein in macrogametes/zygotes. Interestingly, the GLA-Alum combination adjuvant was the most potent inducer of TB antibodies based on serum collected after two immunizations. In agreement with previous observations, biological activity in the SMFA correlated well with the level of anti-Pfs48/45 antibodies.
\end{abstract}

Conclusion: The combined data provide a strong basis for entering the next phase of clinical grade R0.10C production and testing.

Keywords: Plasmodium falciparum malaria, R0.10C immunogenicity, Alhydrogel, Glucopyranosyl Lipid Adjuvant (GLA), Pfs48/45 vaccine candidate, Standard Membrane Feeding Assay (SMFA)

\footnotetext{
*Correspondence: will.roeffen@radboudumc.nl

1 Department of Medical Microbiology, Radboud University Medical

Center, Nijmegen, The Netherlands

Full list of author information is available at the end of the article
} 


\section{Background}

Malaria is primarily confined to the poorest tropical areas of the world and constitutes one of the world's greatest public health problems [1]. Efforts to eliminate malaria transmission through use of insecticides and/or bed nets have resulted in only limited success [2]. Vaccines hold the potential for effective control and elimination of the disease burden. However, despite decades of research there is still no clinically applicable vaccine available. With the renewed focus on malaria eradication, transmission blocking malaria vaccines (TBMV) which induce antibodies that target the sexual stages of the parasite have become a focus of malaria vaccine research. One of the leading candidates for a TBMV is $P f s 48 / 45$, a protein expressed in gametocytes and on the surface of gametes and zygotes [3-6]. Pfs48/45 is essential for male gamete fertility in Plasmodium [7] and anti-Pfs48/45 antibodies have been found to prevent zygote development in the standard membrane feeding assay (SMFA) [8]. The exact mechanism through which these antibodies interfere with fertility is unknown but steric hindrance is likely a possibility. Irrespective of the mechanism, it has been shown in ex vivo assays that there is a strong correlation between levels of $P f$ s $48 / 45$-specific antibodies in sera from malaria-endemic areas and TB activity in the SMFA [9-11].

$P f_{s} 48 / 45$ is a relatively cysteine-rich protein with multiple disulfide bonds resulting in antibody epitopes that are dependent on tertiary structure rather than linear amino acid sequence. A panel of mAbs have been generated and characterized with respect to their TB-activity in SMFA $[12,13]$. Of these, mAb 85RF45.1 exhibits the strongest TB activity whereas mAbs 85RF45.2 and 85RF45.3 do not exhibit TB activity on their own but may act synergistically with 85RF45.1 [12]. All three mAbs recognize conformational epitopes, of which epitope I, defined by mAb 85RF45.1, is located in the C-terminal portion of Pfs48/45 (10C) [14]. Pfs $48 / 45$ and fragments thereof has been produced on recombinant form in various expression systems [5, 6, 15-18]; however, none of these products has reached clinical development phase. Recently, correctly folded fragments were produced of $P f_{\mathrm{s}} 48 / 45$ $(10 \mathrm{C})$ containing all three epitopes and $P f s 48 / 45$ (6C) containing only TB-epitope 1 fused in frame with the $\mathrm{N}$-terminal part of the glutamate rich protein (GLURP) in the Lactococcus lactis expression system [19, 20]. This system has previously proved efficient for the production of GMZ2, an asexual blood-stage vaccine candidate $[21,22]$, and GMZ2 adjuvanted in $\mathrm{Al}(\mathrm{OH})_{3}$ has shown good safety and tolerability in phase 1 clinical trials [21, 23-25].

With the aim of developing a TBMV, the effects of different adjuvant formulations were investigated in mice and rats on: (1) the production of antigen-specific IgG against recombinant and native proteins; and, (2) the biological activity of antibodies elicited by vaccination. The adjuvant vehicles used were either aluminium hydroxide (Alum), an oil-in-water stable emulsion (SE) or $\mathrm{AbISCO}^{\circledR}-100$, a saponin based adjuvant. Two of these formulations (Alum and SE) were supplemented with the Toll-like receptor synthetic TLR 4 agonist glucopyranosyl lipid A adjuvant (GLA) [26]. GLA was used in the present study because it has been shown to enhance IgG responses against the GLURP.R0 component of GMZ2 in mice [27], and GLA is safe for use in humans and nonhuman primates [28].

\section{Methods}

\section{Construction, fermentation and purification of correctly folded R0.10C}

Construction of the recombinant R0.10C hybrid molecule, a fusion protein containing the regions GLURP ${ }_{27-500}$ and $P f \mathrm{~s} 48 / 45_{159-428}$ and a stretch of six histidines in the C-terminus, has been described in detail elsewhere [19]. The theoretical molecular weight of the protein is $89.8 \mathrm{kDa}$ of which $30.7 \mathrm{kDa}$ originates for the $10 \mathrm{C}$ fragment.

All fermentations at the 40L scale were done at the cGMP-facility of Gennova, India, using the standard proposed medium composition as described earlier [19]. Briefly, a fermentor containing reconstituted medium with the final $\mathrm{pH} 7.0$, adjusted with sterile $2 \mathrm{~N} \mathrm{NaOH}$, was inoculated with overnight culture of inoculum. The fermenter was programmed to maintain $\mathrm{pH}$ 6.5, temperature $30{ }^{\circ} \mathrm{C}$ at the low agitation rate $(100 \mathrm{rpm})$ without aeration, maintaining anaerobic conditions. At the stationary phase when no more alkali $(2 \mathrm{~N} \mathrm{NaOH})$ uptake was observed to maintain $\mathrm{pH} 6.5$, the culture was harvested from the fermenter. Following clarification of the fermentation broth by centrifugation, the culture supernatant was concentrated five to six times using tangential flow filtration (TFF) and diafiltered using $2.5 \mathrm{sq} \mathrm{m}$ membrane with pore size cut-off of $10 \mathrm{kDa}$ with buffer- 1 (50 mM phosphate buffer, $\mathrm{pH} 7.0,6$ diafiltration volumes (DV) followed by buffer-2 (50 mM phosphate buffer, $\mathrm{pH} 7.0$ and $0.25 \mathrm{M} \mathrm{NaCl}, 6 \mathrm{DV})$. The diafiltrate, now defined as 'affinity-input' containing $10 \mathrm{mM}$ imidazole and $5 \mathrm{mM} \beta$-ME, was loaded on the Ni2+-Sepharose column pre-equilibrated with $4 \mathrm{CV}$ of equilibration buffer (50 mM phosphate buffer, pH 7.0, $0.25 \mathrm{M} \mathrm{NaCl}$, $20 \mathrm{mM}$ Imidazole). After loading affinity-input, the column was washed with 4 column volumes $(\mathrm{CV})$ of equilibration buffer, followed by washing with redox-buffer of glutathione exists in both $4 \mathrm{mM}$ reduced $(\mathrm{GSH})$ and $0.4 \mathrm{mM}$ oxidized (GSSG) states for $60 \mathrm{~min}$. Following redox-wash, the recombinant $\mathrm{R} 0.10 \mathrm{C}$ was eluted isocratically with the elution buffer containing $50 \mathrm{mM}$ phosphate 
buffer, $0.25 \mathrm{M} \mathrm{NaCl}, 200 \mathrm{mM}$ Imidazole, pH 7.0. The peak fraction was finally purified on anti-Pfs $48 / 45 \mathrm{mAb}$ 45.1 (epitope 1) coupled to agarose using the AminoLink $^{\circledR}$ Plus Immobilization Kit (Pierce: 44894) according to the instructions of the manufacturers. The eluate contained immune purified R0.10C (correctly folded) whereas the run-through contained $<1 \%$ of correctly folded R0.10C. Eluted samples were concentrated to at least $1 \mathrm{mg} / \mathrm{ml}$ against $1 \times$ phosphate buffered saline (PBS) on a Vivaspin20, 30-kDa molecular mass cut-off ultrafiltration unit (Vivascience, Germany). Protein concentrations were measured on a NanoDrop 2000 (Thermo Scientific, USA) using a molar extinction coefficient of 0.3 and Bradford's method.

\section{Rat and mouse immunogenicity studies}

Experimental animals, including female Wistar Hanover rats and female CB6F1 (BALB/c $\times$ C57BL/6 F1 hybrid) mice, were selected for the study. The animals were purchased from Charles River and kept in the Laboratory Animal Facility Centre of the Radboud University Medical Centre (RUMC). All procedures regarding animal immunization complied with European and national regulations.

Immunizations were performed by the subcutaneous route three times, 2 weeks apart with $20 \mu \mathrm{g}$ of R0.10C adjuvanted with different formulations in a total volume of $100 \mu \mathrm{l}$ for mice and $200 \mu \mathrm{l}$ for rats.

Rats receiving adjuvant formulations aluminium hydroxide [(Alhydrogel, Brenntag (Alum: SSI (expiry date June 2012)], Alum/GLA, SE/GLA and AbISCO-100 and for mice complete Freund's adjuvant (CFA)/incomplete Freund's adjuvant (IFA) (Sigma-Aldrich), Alum/GLA, SE/GLA and AbISCO-100 (ISCONOVA). The Freund's group was used for reasons of comparison with the former MBP-PF10C immunizations previously described [6]. For the Alum-based formulation, groups of five animals were immunized with R0.10C adsorbed to Alum with or without a supplement of $5 \mu \mathrm{g}$ of an aqueous formulation of GLA (IDRI:AQ014). For the SE-based immunization, groups of five animals were immunized with R0.10C and SE (IDRI:EM030) supplemented with $5 \mu \mathrm{g}$ of GLA in SE (IDRI:EM031). The components of the Alum and SEbased formulations were mixed gently for $30 \mathrm{~min}$ and allowed to rest on ice for $1 \mathrm{~h}$ before injection. Sera were collected from peripheral blood samples on days $0,14,28$, and 42 separated by centrifugation of blood at $1500 \times g$ for $10 \mathrm{~min}$ and stored at $-20^{\circ} \mathrm{C}$ until further analysis.

\section{ELISA}

Antibody responses in immunized animals were measured by ELISA. All experimental steps, except for coating, were performed at room temperature (RT). Briefly,
ELISA MaxiSorp microtitre plates (Sterilin ${ }^{\circledR}$ ELISA plates, The Netherlands) were coated at $4{ }^{\circ} \mathrm{C}$ overnight with $50 \mu \mathrm{l}$ of the R0.10C $(2 \mu \mathrm{g} / \mathrm{ml})$, GLURP.RO $(1.4 \mu \mathrm{g} /$ $\mathrm{ml})$ or MBP.10C $(2 \mu \mathrm{g} / \mathrm{ml})$ in PBS pH 7.4. Wells were then blocked with $5 \%$ skimmed milk powder in PBS for $1 \mathrm{~h}$ followed by three washings with PBS containing $0.05 \%$ Tween-20 (PBST). Plates were incubated with $50 \mu \mathrm{L}$ twofold serial dilutions of sera starting with 1:100 in PBST for $4 \mathrm{~h}$ at RT. After washing, the plates were incubated with $50 \mu \mathrm{L}$ of $1: 3000$ diluted rabbit-anti rat IgG-HRP $(\mathrm{H}+\mathrm{L})$ (DAKO, 1:3,000 diluted in PBST) or rabbit antimouse IgG-HRP (DAKO, 1:4,000 diluted in PBST) for $2 \mathrm{~h}$. Wells were washed with PBS and subsequently incubated with tetramethyl benzidine (TMB) substrate solution for $20 \mathrm{~min}$. The colour reaction was stopped with $0.2 \mathrm{~N} \mathrm{H}_{2} \mathrm{SO}_{4}$, and the optical density (OD) was read at $450 \mathrm{~nm}$ in an Anthos 2001 Microplate Reader (Labtec BV). Midpoint (EC50) values were calculated using GraphPad Prism, (GraphPad Software, USA). MBP.10C is used for comparison of results with the data as described by Outchkourov et al. [6]. The discrepancy between the antibody titer measured in the R0.10C and MBP.10C ELISAs is due to antibodies against the R0 region of R0.10C.

\section{Standard membrane feeding assay (SMFA)}

Antisera obtained from mice and rats immunized with R0.10C were assessed for TB activity by SMFA as previously described [29,30]. Briefly, $30 \mu \mathrm{l}$ of rat or mouse serum was mixed with $90 \mu \mathrm{l}$ of naïve human serum and $150 \mu \mathrm{l}$ of in vitro gametocyte cultures of the $P$. falciparum (NF54) and for comparison (NF135.C10 Cambodian line [31]) laboratory lines. The mixture was fed to Anopheles stephensi mosquitoes through a membrane feeding apparatus. Pre-immune sera served as the controls. Fully engorged mosquitoes were separated and held at $26{ }^{\circ} \mathrm{C}$. Seven days later, midguts of 20 mosquitoes were examined for oocysts. The observed TB activity of serum (TRA) was determined as the percentage reduction in the arithmetic mean oocyst number in test samples compared to that in controls. The experiment was considered valid when at least $85 \%$ of the mosquitoes feeding on control sera were infected. Data were analysed by a nonparametric test by comparing the medians of two groups using the Mann-Whitney test, or by comparing three or more groups using the Kruskal-Wallis test, followed by post-tests. If significance was indicated, Dunn's analysis was used for comparison with the control group.

\section{Results}

Purification of properly folded R0.10C

Recently, a correctly folded fragment was produced of $P f \mathrm{~s} 48 / 45$ (10C) as a chimera (R0.10C) fused in frame with a section of the $P$. falciparum glutamate rich protein 
(GLURP.R0) in the Lactococcus lactis expression system [19]. R0.10C was immune-purified on mAb 85RF45.1 bound to agarose beads. The resulting immune-purified R0.10C reacted strongly with mAbs against the conformational epitopes $1,2 \mathrm{~b}$ and 3 as demonstrated by sandwich ELISA (Fig. 1a) suggesting that this preparation contains a homogeneous solution of correctly folded R0.10C. In contrast, the flow-through (FT) did not show an appreciable binding to any of the mAbs (Fig. 1a-c) and only affinity-purified R0.10C showed strong reactivity with mAb 85 RF 45.1 by Western blotting (Fig. 1c). To establish a standard reference curve for estimating the percentage of correctly folded protein species in a solution, immune-purified R0.10C (termed $100 \%$ correctly folded) was mixed with the FT containing only misfolded antigen to obtain mixtures of $100,75,50,25,12,6.5$, and $<1 \%$, correctly folded R0.10C, respectively. When each mixture was tested in the sandwich ELISA, there was a strong correlation between the EC50 values and percentage of properly folded antigen (Fig. 1d, $r=0.99$ ). Using this reference, the Ni-IMAC purification step yields a product which contains approximately $5 \%$ correctly folded conformers (Fig. 1d).

\section{Immunogenicity of properly folded R0.10C}

In order to study the immunogenicity of R0.10C, different adjuvants formulations were investigated in both mice and rats. Inbred CB6F1 (BALB/c x C57BL/6 F1 hybrid) mice were immunized three times at two-week intervals with $20 \mu \mathrm{g}$ of immune-purified R0.10C administered in AbISCO, Alum supplemented with GLA, or in an oilin-water SE supplemented with GLA. For comparison, a control group received R0.10C in Freund's adjuvant. Sexual blood-stage, specific antibody responses were measured by ELISA. Two weeks after the last injection (day 42), all mice had relatively high antibody titres against the
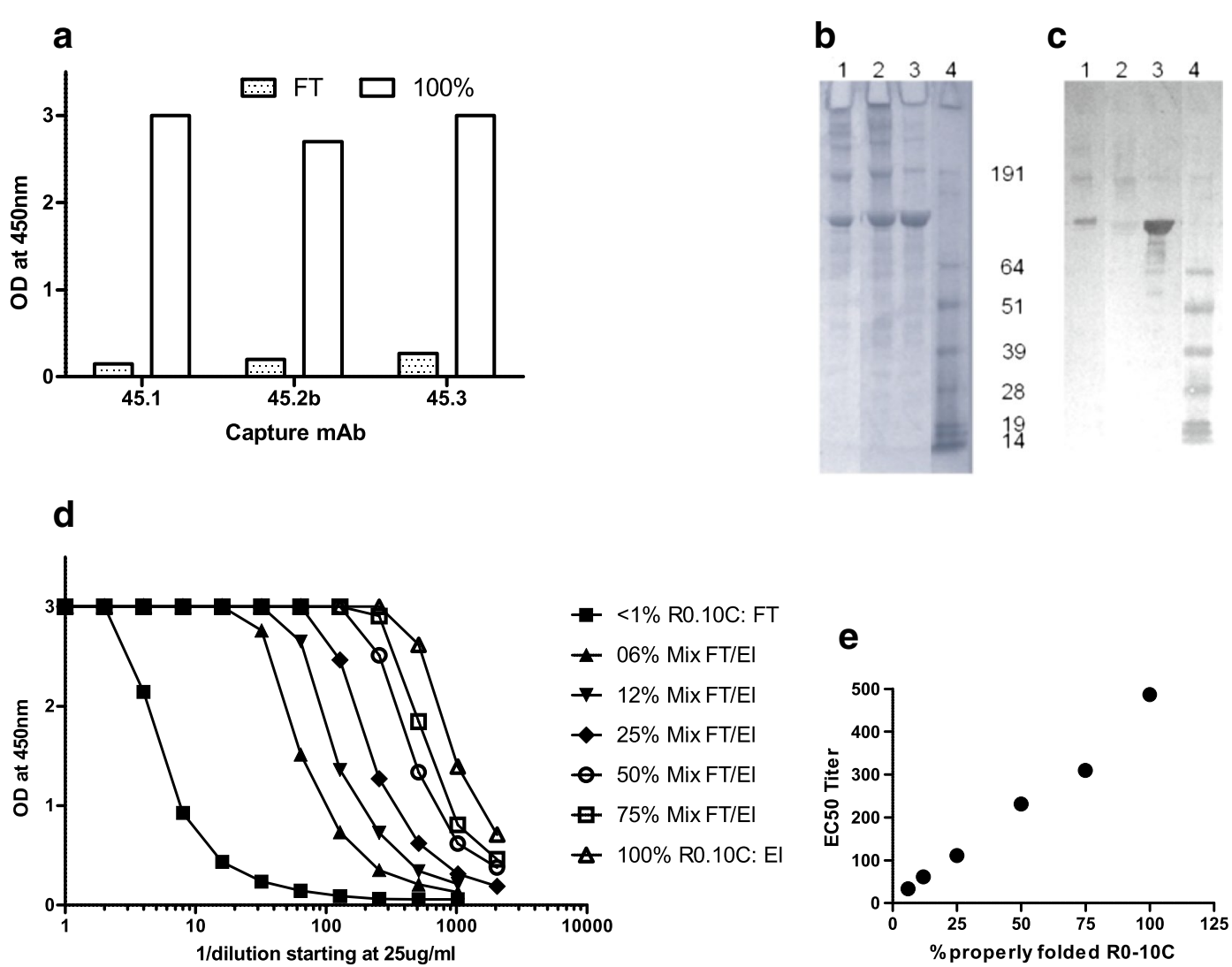

Fig. 1 RO-10C purified by mAb45.1-affinity chromatography. Purification and characterization of correctly folded monomeric R0.10C. a 2-site ELISA with the flow trough (FT) and immune purified antigen (500 ng/well) using mAb 85RF45.1, 85RF45.2b and 85RF45.3 as capture and anti-HIS-HRPO for detection. b Coomassie blue-stained 4-12\% Bis-Tris gel of R0.10C purification steps using $5 \mu \mathrm{g}$ antigen/lane, c Western blot analysis using rat mAb 85RF45.1-HRP for detection. Lane 1, input antigen after $\mathrm{Ni}^{2++}$ column purification; Lane 2, Flow trough (FT: misfolded R0.10C) from the mAb Affinity column: lane 3, eluate (immune purified R0.10C (properly folded)) from the mAb affinity column; Lane 4, Markers, the sizes (kDa) of the molecular mass markers are indicated. $\mathbf{d}$ 2-site ELISA with the FT, mAb-Affinity eluted antigen (EI) and mixed samples using mAb 85 RF45.1 as capture and mAb 85RF45.2b-HRPO for detection. e correlation between EC50 titres and percentage of properly folded antigen ( $R=0.9944$ : $y=4.59 x+0.505)$ 
immunogen (Fig. 2a). When assessing the IgG responses against the $P f_{\mathrm{s}} 48 / 45$ components, it was evident that R0.10C in SE supplemented with GLA gave the lowest and significantly different $(\mathrm{p}=0.016)$ anti-Pfs $48 / 45$ IgG response compared to the control group (Fig. 2b). In contrast, $\mathrm{R} 0.10 \mathrm{C}$ in Alum supplemented with GLA gave a relatively high anti-Pfs48/45 IgG response indistinguishable $(\mathrm{p}=0.3)$ from the response obtained with R0.10C in Freund's adjuvant (Fig. 2b). The finding that an Alum-based formulation is as efficient as an oil-in-water emulsion was unexpected and warrants further investigation. A second experiment, including an additional group of R0.10C/Alum, was therefore performed in rats. Rats were chosen partly to confirm the initial finding in a second species and partly because rats can donate more blood, allowing analysis of individual samples in functional bioassays. Animals were immunized three times with $20 \mu \mathrm{g}$ immune purified R0.10C adjuvanted in Alum, Alum/GLA, SE/GLA or AblSCO (Fig. 2c). Compared with the mouse experiment, R0.10C adjuvanted in Alum/GLA gave the lowest anti-10C IgG levels and is significantly different from the Alum group $(\mathrm{p}=0.008)$. Furthermore, R0.10C adjuvanted in Alum gave the highest anti-Pfs48/45 IgG response and is also significantly different from the SE/GLA group $(\mathrm{p}<0.01)$ but not from the AbISCO group ( $p>0.2$ ) (Fig. 2d).

Antibodies against GLURP.R0 showed that the lowest antibody titers (EC50 titer $=1984$ with standard deviation (SD) of 889) was detected for the alum group but is only significantly different $(\mathrm{p}=0.01)$ from the SE/GLA and AbISCO group. There was no differences in antibody titers between the Alum/GLA, SE/GLA and AbISCO
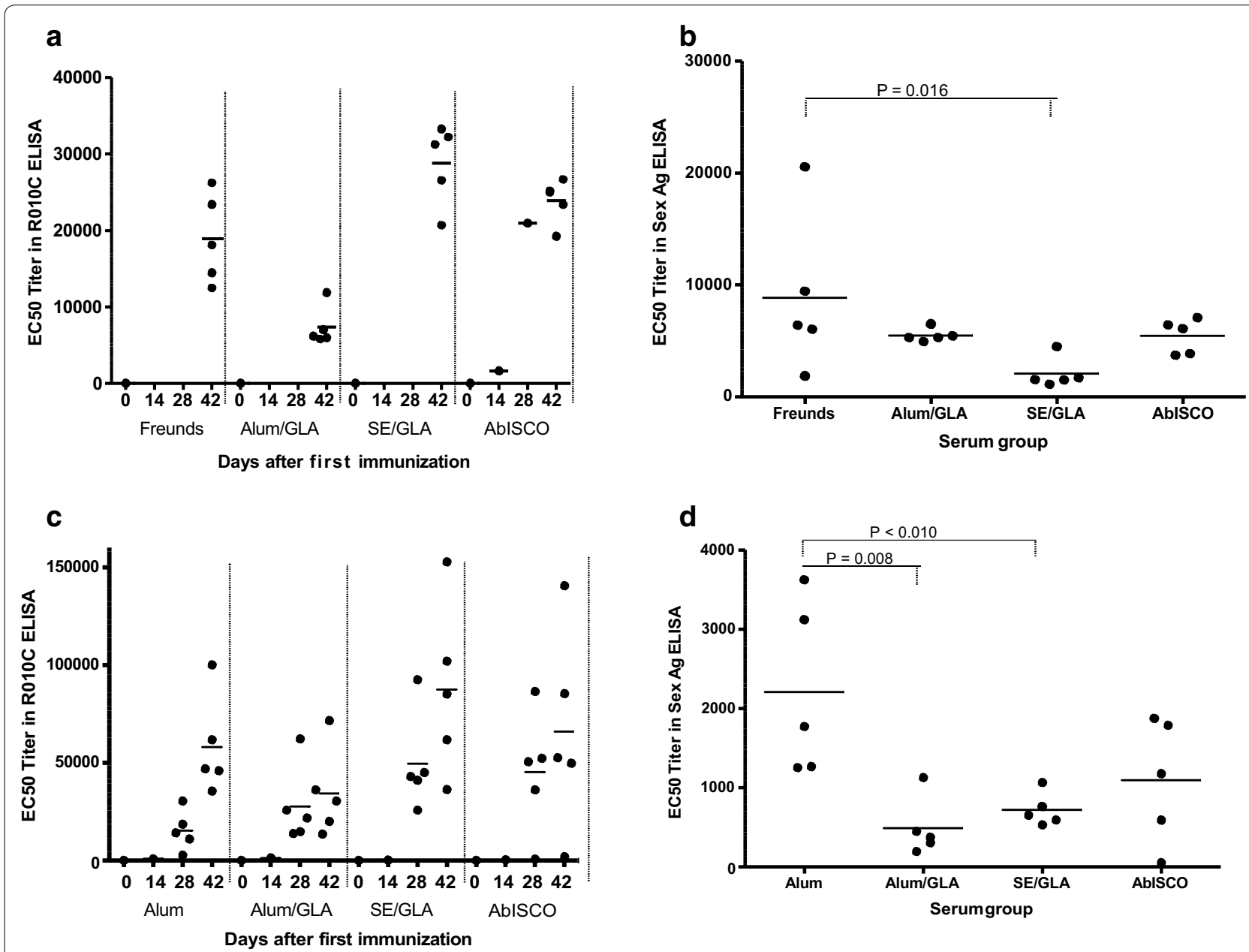

Fig. 2 Immunogenicity of R0.10C. ELISA results of various bleed sera from mice (a b upper graphs) and rats (c, $\mathbf{d}$ lower graphs) immunized with R0.10C and different adjuvants. 0, 14, 28, and 42 indicate pre-immune sera, day 14, day 28 and day 42 of the time course of the immunization for mouse (a) and rat (c). Mouse sera (a) from day 14 and 28 were not available. The right figures are ELISA results of final bleed (day 42 ) sera from mice (b) and rats (d) immunized with immune purified R0.10C formulated with different adjuvants using 10C as sexual stage antigen (Sex Ag) 
group; EC50 titers $4776(\mathrm{SD}=2419), 6371(\mathrm{SD}=4093)$ and $7462(\mathrm{SD}=4098)$, respectively.

\section{Inhibition of sexual Plasmodium falciparum fertilization by R0.10C antisera}

TB activity of anti-R0.10C antisera was assessed in the SMFA. The pre-bleed (day 0 ) of mice and rats did not show any $\mathrm{TB}$ activity and was used to calculate the per cent inhibition of oocyst counts. Two injections of R0.10C formulated in Alum/GLA or AbISCO elicit IgG levels which efficiently inhibited (>96\%) oocyst development, whereas three injections of R0.10C in SE/GLA and Freund's were required to reach the same level of inhibition (Table 1). The same trend was also observed in rats (Table 1).

When testing individual day 42 sera from rats, the TB activity of the four adjuvant formulations resulted in $>99 \%$ inhibition of oocyst development except for one rat in the Alum/GLA (TRA $25 \%$ ) and one in the AbISCO group (TRA $29 \%$ ) (Table 2). These two non-blocking antisera also gave the lowest anti-Pfs48/45 IgG titres (196 and 51 respectively).

\section{R0.10C antisera inhibit Plasmodium falciparum growth in a} strain independent manner

Antigenic variation may limit the breadth of $P$. falciparum-inhibiting antibodies. To gauge the breadth of $P$. falciparum inhibition antisera from all animals immunized with R0.10C/Alum was tested for TB activity of genetically diverse African and Cambodian P. falciparum laboratory lines NF54 and NF135.10C, respectively. The individual antisera showed comparable levels of TB activity (Table 3 ) for the two strains irrespective of polymorphism at amino acid positions 254, 304 and 322 in the Pfs $48 / 45$ portion of R0.10C, suggesting that anti-R0.10C IgG act in a strain-independent manner to control parasite fertility in the infected mosquito. Furthermore, the $\mathrm{TB}$ activity for the alum-immunized rats is higher
(76-100 \%; EC50 titers: 1250-3622) compared with the alum/GLA group (0-72 \%; EC50 titers: 196-446) tested at a dilution of $1 / 27$ for both strains.

\section{Discussion}

The development of a TBMV is an attractive goal because it holds the potential to accelerate elimination or even eradication of $P$. falciparum malaria. The inclusion of a TBMV in these efforts would require a highly immunogenic vaccine formulation with the capacity to induce high levels of TB antibodies lasting for at least one transmission season. This is particularly important since several malaria antigens have proved to be poorly immunogenic in adjuvants for human use [32, 33]. Recently, a new multi-stage vaccine candidate (R0.10C) was developed consisting of the N-terminal domain of GLURP (R0) fused in frame to a functional domain of $P f s 48 / 45$ (10C) [19]. The potential importance of increasing the overall immunogenicity of $\mathrm{R} 0.10 \mathrm{C}$ and the $P f s 48 / 45$ domain in particular, is suggested by the direct relationship between anti-R0.10C antibody titre and biological activity in the SMFA [19]. In the present study, a series of adjuvant formulations in small rodents were investigated for their ability to enhance antibody responses against sexual blood stages. Antisera were also investigated for their ability to control parasite fertilization in the SMFA. It is evident that both Alum and SE/GLA formulations elicited strong TB immunity with five out of five animals showing $>99 \%$ TRA. In contrast, R0.10C formulated in Alum/GLA or in AbISCO was slightly less efficient with four out of five animals seroconverting to functional antibody titres. All mice and rat sera were tested at a 1/9 dilution. Testing rat sera from the Alum and Alum/GLA group at dilution of $1 / 27$ still resulted in strong TB activity for the Alum group but not for the Alum/GLA group, showing that Alum alone is a better adjuvant for functional antibodies.

Table 1 Infectivity of $P$. falciparum Gametocytes to A. stephensi Mosquitoes in the presence of different pooled bleed sera from mice and rats immunized with R0.10C formulated with different adjuvants in the Standard Membrane-Feeding Assay (SMFA)

\begin{tabular}{|c|c|c|c|c|c|c|c|c|c|c|c|c|c|c|c|c|}
\hline \multirow[t]{3}{*}{ Day } & \multicolumn{8}{|c|}{ Mice } & \multicolumn{8}{|c|}{ Rats } \\
\hline & \multicolumn{2}{|c|}{ Freunds } & \multicolumn{2}{|c|}{ Alum/GLA } & \multicolumn{2}{|c|}{ SE/GLA } & \multicolumn{2}{|c|}{ AbISCO } & \multicolumn{2}{|c|}{ Alum } & \multicolumn{2}{|c|}{ Alum/GLA } & \multicolumn{2}{|c|}{ SE/GLA } & \multicolumn{2}{|c|}{ AbISCO } \\
\hline & Inf & TRA, \% & Inf & TRA, \% & Inf & TRA, \% & Inf & TRA, \% & Inf & TRA, \% & Inf & TRA, \% & Inf & TRA, \% & $\operatorname{lnf}$ & TRA, \% \\
\hline 0 & 18 & & 18 & & 19 & & 18 & & 16 & & 17 & & 19 & & 16 & \\
\hline 14 & 17 & 0 & 11 & 35 & 11 & 26 & 18 & 0 & 20 & 11 & 17 & 2 & 20 & 0 & 17 & 0 \\
\hline 28 & 7 & 76 & 1 & 97 & 10 & 59 & 4 & 96 & 5 & 94 & 3 & 97 & 10 & 85 & 6 & 91 \\
\hline 42 & 0 & 100 & 2 & 95 & 1 & 99 & 1 & 99 & 1 & 98 & 1 & 99 & 1 & 99 & 1 & 99 \\
\hline
\end{tabular}

Pooled Sera (1:9 diluted) from the different bleeds (Day 0, 14, 28 and 42) were tested in the SMFA. Seven days after membrane feed, the number of infected mosquitoes and oocyst density were determined. Inf: total infected mosquitoes from the 20 dissected mosquitoes; TRA, \%: percentage of Transmission Reducing Activity using the pre-bleed (Day 0 ) sample for calculation 
Table 2 Infectivity of $P$. falciparum Gametocytes to $A$. stephensi Mosquitoes in the presence of day 42 serum from rat immunized with $\mathrm{RO} .10 \mathrm{C}$ formulated with different adjuvant formulation in the Standard Membrane Feeding Assay (SMFA

\begin{tabular}{|c|c|c|c|c|c|}
\hline Group & Rat & Inf & Mean (SD) & $\%$ & $P$ value \\
\hline \multirow[t]{5}{*}{ Alum } & 1 & 1 & $0.05(0.22)$ & 99 & $<0.001$ \\
\hline & 2 & 0 & $0.0(0.0)$ & 100 & $<0.001$ \\
\hline & 3 & 0 & $0.0(0.0)$ & 100 & $<0.001$ \\
\hline & 4 & 1 & $0.05(0.22)$ & 99 & $<0.001$ \\
\hline & 5 & 0 & $0.0(0.0)$ & 100 & $<0.001$ \\
\hline \multirow[t]{5}{*}{ Alum/GLA } & 6 & 0 & $0.0(0.0)$ & 100 & $<0.001$ \\
\hline & 7 & 1 & $0.05(0.22)$ & 99 & $<0.001$ \\
\hline & 8 & 18 & $7.85(4.64)$ & 25 & NS \\
\hline & 9 & 1 & $0.05(0.22)$ & 99 & $<0.001$ \\
\hline & 10 & 1 & $0.05(0.22)$ & 99 & $<0.001$ \\
\hline Pre sera & & $37 / 40$ & $10.48(6.12)$ & & \\
\hline \multirow[t]{5}{*}{ SE/GLA } & 11 & 2 & $0.10(0.31)$ & 99 & $<0.001$ \\
\hline & 12 & 2 & $0.10(0.31)$ & 99 & $<0.001$ \\
\hline & 13 & 1 & $0.05(0.22)$ & 99 & $<0.001$ \\
\hline & 14 & 1 & $0.05(0.22)$ & 99 & $<0.001$ \\
\hline & 15 & 0 & $0.0(0.0)$ & 100 & $<0.001$ \\
\hline \multirow[t]{5}{*}{ AblsCO } & 16 & 0 & $0.0(0.0)$ & 100 & $<0.001$ \\
\hline & 17 & 16 & 7.45 (6.39) & 29 & NS \\
\hline & 18 & 0 & $0.0(0.0)$ & 100 & $<0.001$ \\
\hline & 19 & 1 & $0.05(0.22)$ & 99 & $<0.001$ \\
\hline & 20 & 2 & $0.10(0.31)$ & 99 & $<0.001$ \\
\hline
\end{tabular}

Sera (1:9 diluted) were tested in the SMFA. Seven days after membrane feed, the number of infected mosquitoes and oocyst density were determined. Sera: Rat serum from R0.10C-immunized group; Control: feeder with pre-bleed serum (day 0 ) of the group; no. Inf: total infected from 20 dissected mosquitoes; TRA, \%: percentage of Transmission Reducing Activity; P-value: sample of the control (day 0) was used for calculation

NS not significant; SD standard deviation
The analysis of specific antibody responses against the $10 \mathrm{C}$ domains of R0.10C suggests that the Alum formulation lead to the highest levels of 10C-specific antibodies compared to all other formulations (Fig. 2d). In contrast, an oil-in-water emulsion formulation of GLA led to relatively lower levels of 10C-specific IgG in mice, but not in rats (Fig. 2b). The discrepancy between the antibody titer measured in the R0.10C and MBP.10C ELISAs is due to antibodies against the R0 region of R0.10C. Since recombinant MBP.10C only share the Pfs48/45$10 \mathrm{C}$ region with the $\mathrm{R} 0.10 \mathrm{C}$ fusion protein, MBP.10C was used for the detection of 10C-specific antibodies. MBP.10C was included in the present study to allow comparison with previously published data [6]. Re-testing in a larger group of animals and/or using the $10 \mathrm{C}$ part alone may help to clarify whether there is indeed a specific difference.

The identification and characterization of TB epitopes of $P f s 48 / 45$ have been facilitated by the availability of mAbs with TB activity $[8,12,13]$. In the process of developing a $P f \mathrm{~s} 48 / 45$-based TBMV, a series of mAbs was used against conformation-dependent epitopes for the characterization of recombinant $P f s 48 / 45[6,13]$. Here, it has demonstrated that a R0.10C antigen preparation which is correctly folded with respect to epitope 1 of $P f s 48 / 45$ elicits high levels of antibodies against this epitope in rats with the capacity to inhibit parasite growth in the mosquito when adjuvanted in Alum, Alum/GLA, SE/GLA, or AbISCO. Sera which inhibit a high level of TB activity (TRA) also has a low mosquito infection rate (Tables $1,2,3$ ). Adjuvanted with Alum resulted in the highest TB activity (Table 3). Recently, Singh and others [20] show that R0.6C (only epitope 1

Table 3 Comparison of percentage Transmission-Reducing Activity using 2 Pf strains of different geographical origin and sera from rats immunized with R0.10C Alum and Alum/GLA formulation

\begin{tabular}{|c|c|c|c|c|c|c|c|}
\hline \multirow[t]{2}{*}{ Group } & \multirow[t]{2}{*}{ Rat } & \multicolumn{3}{|l|}{ NF54 } & \multicolumn{3}{|c|}{ NF135 } \\
\hline & & Inf & Mean (SD) & TRA, \% & Inf & Mean (SD) & $\begin{array}{l}\text { TRA, } \\
\%\end{array}$ \\
\hline \multirow[t]{5}{*}{ Alum } & 1 & 16 & $7.35(6.23)$ & 82 & 17 & $6.60(5.99)$ & 76 \\
\hline & 2 & 17 & $4.45(4.00)$ & 89 & 6 & $0.60(1.14)$ & 98 \\
\hline & 3 & 1 & $0.05(0.22)$ & 100 & 0 & $0.0(0.0)$ & 100 \\
\hline & 4 & 16 & $4.30(4.71)$ & 89 & 10 & $0.90(1.21)$ & 97 \\
\hline & 5 & 17 & $3.20(2.86)$ & 92 & 4 & $0.39(0.78)$ & 99 \\
\hline \multirow[t]{4}{*}{ Alum/GLA } & 6 & 18 & $27.7(14.7)$ & 31 & 17 & $7.70(6.87)$ & 72 \\
\hline & 7 & 19 & $38.8(23.3)$ & 3 & 20 & $15.4(9.2)$ & 45 \\
\hline & 8 & 20 & $41.7(25.9)$ & 0 & 18 & $27.1(15.2)$ & 3 \\
\hline & 9 & 20 & $17.8(8.1)$ & 55 & 18 & $10.0(5.85)$ & 64 \\
\hline Pre sera & & $35 / 35$ & 39.9 (23.6) & 0 & $40 / 40$ & $27.8(12.6)$ & 0 \\
\hline
\end{tabular}

Day 42 sera (1:27 diluted) were tested in the SMFA. Seven days after membrane feed, the number of infected mosquitoes and oocyst density were determined. Sera: rat serum from R0.10C-immunized group; Control: feeder with pooled pre-bleed serum (day 0 ) of the group. Inf: total infected from 20 dissected mosquitoes; SD: Standard deviation; TRA, \%: percentage of Transmission Reducing Activity. NF54: African strain and NF153: Cambodian strain 
of $P f s 48 / 45)$ did also give high TB activity. Further studies must be performed combining R0.10C and R0.6C for immunogenicity data.

The adjuvants tested here (Alum and SE with/without addition of GLA or AbISCO) are all applicable for human use $[28,34]$. Aluminium salts are the most commonly used adjuvants in clinical trials and they have the reputation of being safe and give high antibody titres and long-lasting antibody responses [34]. Whether R0.10C/ Alum is also a strong immunogen for induction of functional antibodies in humans remains to be determined.

In summary, the Lactococcus lactis expression platform was used for production of correctly folded $P f \mathrm{~s} 48 / 45$. Since Alum has been used in past malaria vaccine trials of GLURP-based vaccine candidates [21, 35], a further vaccination study with the $\mathrm{R} 0.10 \mathrm{C}$ antigen in combination with Alum alone can be pursued.

\section{Conclusions}

An investigation of immune responses to R0.10C using different adjuvants resulted in good antibody response with TB activity. A clinical trial with the R0.10C antigen in combination with Alum appears to be a favourable option.

\section{Authors' contributions \\ WR, MT and RS contributed to the design and performance of the experi- ments and the writing of the manuscript. MvdVB produced the asexual and sexual stage parasites and GVG performed the SMFA. LS, SKS and SK expressed, purified and analysed the recombinant R0.10C protein. TA, GA and $M C$ provided technical advice. All authors have read and approved the final manuscript.}

\section{Author details \\ 1 Department of Medical Microbiology, Radboud University Medical Center, Nijmegen, The Netherlands. ${ }^{2}$ Department for Congenital Disorders, Statens Serum Institut, Copenhagen, Denmark. ${ }^{3}$ Department of International Health, Immunology, and Microbiology, Centre for Medical Parasitology, University of Copenhagen, Copenhagen, Denmark. ${ }^{4}$ Department of Infectious Diseases, Copenhagen University Hospital, Rigshospitalet, Copenhagen, Denmark. \\ ${ }^{5}$ Gennova Biopharmaceuticals Limited, Pune, India.}

\begin{abstract}
Acknowledgements
The aqueous (AQ014) and stable emulsion (EM031) formulations of GLA, as well as stable emulsion alone (EM030), were provided through funding to Steven G Reed (IDRI) from the Bill and Melinda Gates Foundation under grant \#OPP49932. We are very grateful to Randall Howard, IDRI, for help obtaining these formulations and for manuscript review. Thomas Phillipson, ISCONOVA, providing AbISCO adjuvant and SSI for providing Alum adjuvant used in this study. The authors would like to thank Laura Pelser, Jolanda Klaassen, Astrid Pouwelsen and Jacqueline Kuhnen for culturing and dissecting mosquitoes, and Wouter Graumans and Rianne Siebelink-Stoter for parasite culture. The research leading to these results received funding from the European Union Seventh Framework Programme ([FP7/2007-2013] [FP7/2007-2011]) under grant agreement no [242079].
\end{abstract}

\section{Competing interests}

The authors declare that they have no competing interests.

Received: 11 September 2015 Accepted: 27 October 2015

Published online: 09 November 2015

\section{References}

1. WHO: World malaria report 2014. World Health Organization Geneva. Available: http://www.who.int/malaria/publications/world_malaria_ report_2014/en/. 2014.

2. Moiroux N, Gomez MB, Pennetier C, Elanga E, Djenontin A, Chandre $F$, et al. Changes in Anopheles funestus biting behavior following universal coverage of long-lasting insecticidal nets in Benin. J Infect Dis. 2012;206:1622-9.

3. Carter R. Transmission blocking malaria vaccines. Vaccine. 2001;19:2309-14.

4. Sutherland CJ. Surface antigens of Plasmodium falciparum gametocytesa new class of transmission-blocking vaccine targets? Mol Biochem Parasitol. 2009;166:93-8.

5. Chowdhury DR, Angov E, Kariuki T, Kumar N. A potent malaria transmission blocking vaccine based on codon harmonized full length Pfs48/45 expressed in Escherichia coli. PLoS One. 2009;4:e6352.

6. Outchkourov NS, Roeffen W, Kaan A, Jansen J, Luty A, et al. Correctly folded Pfs $48 / 45$ protein of Plasmodium falciparum elicits malaria transmission-blocking immunity in mice. Proc Natl Acad Sci USA. 2008;105:4301-5.

7. van Dijk MR, Janse CJ, Thompson J, Waters AP, Braks JA, Dodemont HJ, et al. A central role for P48/45 in malaria parasite male gamete fertility. Cell. 2001;104:153-64.

8. Vermeulen AN, Roeffen WF, Henderik JB, Ponnudurai T, Beckers PJ, Meuwissen JH. Plasmodium falciparum transmission blocking monoclonal antibodies recognize monovalently expressed epitopes. Dev Biol Stand. 1985;62:91-7.

9. Bousema T, Roeffen W, Meijerink H, Mwerinde H, Mwakalinga S, van Gemert GJ, et al. The dynamics of naturally acquired immune responses to Plasmodium falciparum sexual stage antigens Pfs 230 \& Pfs $48 / 45$ in a low endemic area in Tanzania. PLoS One. 2010;5:e14114.

10. Mulder B, Lensen T, Tchuinkam T, Roeffen W, Verhave JP, Boudin C, Sauerwein R. Plasmodium falciparum: membrane feeding assays and competition ELISAs for the measurement of transmission reduction in sera from Cameroon. Exp Parasitol. 1999;92:81-6.

11. Roeffen W, Mulder B, Teelen K, Bolmer M, Eling W, Targett GA, et al. Association between anti-Pfs $48 / 45$ reactivity and $P$. falciparum transmission-blocking activity in sera from Cameroon. Parasite Immunol. 1996;18:103-9.

12. Vermeulen AN, Ponnudurai T, Beckers PJ, Verhave JP, Smits MA, Meuwissen JH. Sequential expression of antigens on sexual stages of Plasmodium falciparum accessible to transmission-blocking antibodies in the mosquito. J Exp Med. 1985;162:1460-76.

13. Roeffen W, Teelen K, van As J, van de Vegte-Bolmer M, Eling W, Sauerwein R. Plasmodium falciparum: production and characterization of rat monoclonal antibodies specific for the sexual-stage Pfs $48 / 45$ antigen. Exp Parasitol. 2001;97:45-9.

14. Outchkourov N, Vermunt A, Jansen J, Kaan A, Roeffen W, Teelen K, et al. Epitope analysis of the malaria surface antigen $p f s 48 / 45$ identifies a subdomain that elicits transmission blocking antibodies. J Biol Chem. 2007;282:17148-56.

15. Kocken CH, Jansen J, Kaan AM, Beckers PJ, Ponnudurai T, Kaslow DC, et al. Cloning and expression of the gene coding for the transmission blocking target antigen Pfs $48 / 45$ of Plasmodium falciparum. Mol Biochem Parasitol. 1993;61:59-68.

16. Milek RL, DeVries AA, Roeffen WF, Stunnenberg H, Rottier PJ, Konings RN. Plasmodium falciparum: heterologous synthesis of the transmissionblocking vaccine candidate Pfs $48 / 45$ in recombinant vaccinia virusinfected cells. Exp Parasitol. 1998;90:165-74.

17. Milek RL, Stunnenberg HG, Konings RN. Assembly and expression of a synthetic gene encoding the antigen Pfs $48 / 45$ of the human malaria parasite Plasmodium falciparum in yeast. Vaccine. 2000;18:1402-11.

18. Milek RL, Roeffen WF, Kocken CH, Jansen J, Kaan AM, Eling WM, et al. Immunological properties of recombinant proteins of the transmission blocking vaccine candidate, Pfs $48 / 45$, of the human malaria parasite Plasmodium falciparum produced in Escherichia coli. Parasite Immunol. 1998:20:377-85.

19. Theisen $M$, Roeffen W, Singh SK, Andersen G, Amoah L, van de VegteBolmer $\mathrm{M}$, et al. A multi-stage malaria vaccine candidate targeting both transmission and asexual parasite life-cycle stages. Vaccine. 2014;32:2623-30. 
20. Singh SK, Roeffen W, Andersen G, Bousema T, Christiansen M, Sauerwein $R$, et al. A Plasmodium falciparum 48/45 single epitope R0.6C subunit protein elicits high levels of transmission blocking antibodies. Vaccine. 2015:33:1981-6.

21. Esen M, Kremsner PG, Schleucher R, Gassler M, Imoukhuede EB, Imbault $\mathrm{N}$, et al. Safety and immunogenicity of GMZ2-a MSP3-GLURP fusion protein malaria vaccine candidate. Vaccine. 2009;27:6862-8.

22. Theisen M, Soe S, Brunstedt K, Follmann F, Bredmose L, Israelsen H, et al. A Plasmodium falciparum GLURP-MSP3 chimeric protein; expression in Lactococcus lactis, immunogenicity and induction of biologically active antibodies. Vaccine. 2004;22:1188-98.

23. Belard S, Issifou S, Hounkpatin AB, Schaumburg F, Ngoa UA, Esen M, et al A randomized controlled phase $\mathrm{lb}$ trial of the malaria vaccine candidate GMZ2 in African children. PLoS One. 2011;6:e22525.

24. Mordmuller B, Szywon K, Greutelaers B, Esen M, Mewono L, Treut C, et al. Safety and immunogenicity of the malaria vaccine candidate GMZ2 in malaria-exposed, adult individuals from Lambarene. Gabon. Vaccine. 2010;28:6698-703.

25. Esen M, Mordmuller B, de Salazar PM, Adegnika AA, Agnandji ST, Schaumburg $F$, et al. Reduced antibody responses against Plasmodium falciparum vaccine candidate antigens in the presence of Trichuris trichiura. Vaccine. 2012;30:7621-4

26. Baldwin SL, Shaverdian N, Goto Y, Duthie MS, Raman VS, Evers T, et al. Enhanced humoral and Type 1 cellular immune responses with Fluzone adjuvanted with a synthetic TLR4 agonist formulated in an emulsion. Vaccine. 2009;27:5956-63.

27. Lousada-Dietrich S, Jogdand PS, Jepsen S, Pinto VV, Ditlev SB, Christiansen $\mathrm{M}$, et al. A synthetic TLR4 agonist formulated in an emulsion enhances humoral and Type 1 cellular immune responses against GMZ2-a GLURP-MSP3 fusion protein malaria vaccine candidate. Vaccine. 2011;29:3284-92.
28. Coler RN, Duthie MS, Hofmeyer KA, Guderian J, Jayashankar L, Vergara J, et al. From mouse to man: safety, immunogenicity and efficacy of a candidate leishmaniasis vaccine LEISH-F3 + GLA-SE. Clin Transl Immunology. 2015:4:e35.

29. Lensen A, van Druten J, Bolmer M, van Gemert G, Eling W, Sauerwein R. Measurement by membrane feeding of reduction in Plasmodium falciparum transmission induced by endemic sera. Trans R Soc Trop Med Hyg 1996;90:20-2.

30. Ponnudurai T, Lensen AH, Van Gemert GJ, Bensink MP, Bolmer M, Meuwissen JH. Infectivity of cultured Plasmodium falciparum gametocytes to mosquitoes. Parasitology. 1989;98(Pt 2):165-73.

31. Teirlinck AC, Roestenberg M, vandeVegte-Bolmer M, Scholzen A Heinrichs MJ, Siebelink-Stoter R. NF135.C10: a new Plasmodium falciparum clone for controlled human malaria infections. J Infect Dis. 2013;207:656-60.

32. Wu Y, Ellis RD, Shaffer D, Fontes E, Malkin EM, Mahanty S, et al. Phase 1 trial of malaria transmission blocking vaccine candidates Pfs 25 and Pvs25 formulated with montanide ISA 51. PLoS One. 2008;3:e2636.

33. Druilhe P, Spertini F, Soesoe D, Corradin G, Mejia P, Singh S, et al. A malaria vaccine that elicits in humans antibodies able to kill Plasmodium falciparum. PLoS Med. 2005;2:e344

34. Coler RN, Carter D, Friede M, Reed SG. Adjuvants for malaria vaccines. Parasite Immunol. 2009;31:520-8.

35. Hermsen CC, Verhage DF, Telgt DS, Teelen K, Bousema JT, Roestenberg $\mathrm{M}$, et al. Glutamate-rich protein (GLURP) induces antibodies that inhibit in vitro growth of Plasmodium falciparum in a phase 1 malaria vaccine trial. Vaccine. 2007;25:2930-40.

\section{Submit your next manuscript to BioMed Central and take full advantage of:}

- Convenient online submission

- Thorough peer review

- No space constraints or color figure charges

- Immediate publication on acceptance

- Inclusion in PubMed, CAS, Scopus and Google Scholar

- Research which is freely available for redistribution

Submit your manuscript at

www.biomedcentral.com/submit

C Biomed Central 